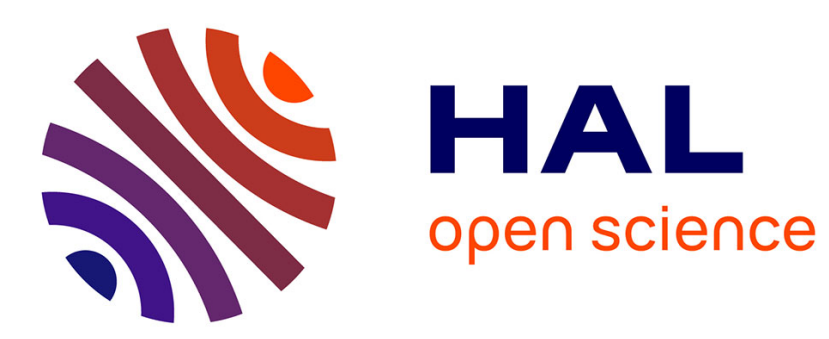

\title{
Transport of small particles through a 3D packing of spheres: experimental and numerical approaches
}

\author{
Franck Lominé, Luc Oger
}

\section{To cite this version:}

Franck Lominé, Luc Oger. Transport of small particles through a 3D packing of spheres: experimental and numerical approaches. Journal of Statistical Mechanics: Theory and Experiment, 2006, 7, pp.P07019. 10.1088/1742-5468/2006/07/P07019 . hal-00145592

\section{HAL Id: hal-00145592 \\ https://hal.science/hal-00145592}

Submitted on 14 Mar 2019

HAL is a multi-disciplinary open access archive for the deposit and dissemination of scientific research documents, whether they are published or not. The documents may come from teaching and research institutions in France or abroad, or from public or private research centers.
L'archive ouverte pluridisciplinaire HAL, est destinée au dépôt et à la diffusion de documents scientifiques de niveau recherche, publiés ou non, émanant des établissements d'enseignement et de recherche français ou étrangers, des laboratoires publics ou privés. 
Postprint of paper published in J Stat Mech Theory Exp.2006; 2006(07): P07019. http://stacks.iop.org/1742-5468/2006/P07019

\title{
Transport of small particles through a 3D packing of spheres: experimental and numerical approaches
}

\author{
Franck Lominé and Luc Oger \\ GMCM, University of Rennes 1, CS 74205, 35042 Rennes, France \\ E-mail: franck.lomine@univ-rennes1.fr, luc.oger@univ-rennes1.fr \\ PACS numbers: 45.70.Mg-81.05.Rm
}

Submitted to: JSTAT

\begin{abstract}
In this paper, we study the transport of particles through a porous structure. Experimentally, we focus our attention on the dependence of the mean transit time on some parameters like the number of small particles injected in the structure, and the height of the packing. We have developed a numerical model, based on a DEM method, to simulate the experiment. This model is useful for accessing the internal structure of the packing and for analysing precisely the influence of the restitution coefficient and the size ratio between spheres.
\end{abstract}

\section{Introduction}

Transport and mixing of granular materials are central features of many industrial processes as in food, chemical engineering, civil engineering, ceramics or pharmaceutical industries. Nevertheless, size segregation, which is a non-random spatial distribution of the different grain species, is often a source of important problems. A well-known example of size segregation effect is 'Brazil nut segregation' [1] also called the Brazil nut effect (BNE). Mixing homogeneously granular media is important but not trivial. One way to succeed is to make grains have a diffusive-like motion. However, in contrast to molecular diffusion, mixing by diffusion of a granular system cannot be achieved without an input of energy. A system that gives grains enough energy to ensure their motion and changes their velocities randomly could be named a granular mixer. Many kinds of mixers are used in the industrial domains previously quoted: rotating drums [2], shakers etc. We are interested in the study of the flow of small particles due to gravity through a packing of large spheres, that build up a sort of porous medium. Such a system could be used for mixing particles of different sizes. But the study of inter-particle percolation of mono-size spheres has to be performed first. When the two grain species are very different in size (with a ratio $D_{\text {large }} / D_{\text {small }} \geq\left(\frac{2}{\sqrt{3}}-1\right)^{-1}=6.464 \ldots$ $[3]$ ), it is possible for the smaller to drain totally through the packing. This kind of 
segregation was previously studied [4] and also more recent studies $[5,6,7]$ have been released. Nevertheless, these cases were limited to launches of one or of a small number of particles at the same time. In this paper, we present results of our experimental and numerical study of inter-particle percolation with large number of grains. In other words, contrary to previous studies, the neighbourhood of spheres is going to influence their individual behaviours. Indeed, the presence of many particles falling together induces many additional collisions compared to the mono-particle case. In the three following sections, we present the experimental set-up and experimental results, and analyse the influence of the number of percolating spheres. Then, in the last section, we present a numerical model that lets us examine in more detail the influence of the different physical parameters.

\section{Experimental set-up}

The experimental set-up, shown in figure 1 , is made of mono-disperse beads packed inside a parallelepiped tank of $26 \mathrm{~cm} \times 26 \mathrm{~cm} \times 51 \mathrm{~cm}$. The packing, constructed under gravity, can be made of spheres of different sizes $D$. It plays the role of a porous medium with a reproducible porosity around 0.4. Another packing made by small iron beads of diameter

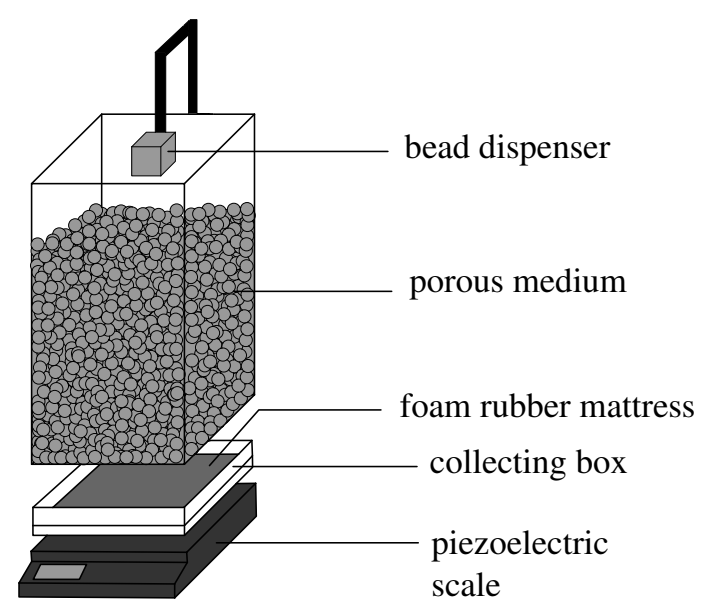

Figure 1. Schematic drawing of the experimental set-up.

$d$ is put in a box placed on a handle, as close as possible to the upper surface. These percolating beads are quasi-instantaneously launched in the porous structure by using a vertical opening hatch. The detection system consists of an electronic weight scale with a piezoelectric material. The signal, from the piezoelectric material, is amplified and taken down by an acquisition card. In order to have reproducible results, the humidity rate (close to 50\%) and the temperature are kept constant. We also use concentric cubes that can be inserted in the bead dispenser with the aim to maintain the launched packing as isotropic (i.e. punctual) as possible.

We work with a ratio far from the trapping threshold in order to free ourselves from trapping events. Indeed, as mentioned previously, a ratio of $\frac{D}{d} \geq 6.464 \ldots$ [3] permits 
any particle to pass through any pores of the structure. It is convenient to estimate the maximum interstitial volume between four spheres of diameter $D$ in a tetrahedral arrangement. This one is the accessible volume for percolating particles of diameter $d$. If we take into account the random close packing limit $\left(\Phi_{\mathrm{RCP}}=0.64\right)$, we can define $V_{p}=\Phi_{\mathrm{RCP}} \frac{D^{3}}{12}(\sqrt{2}-2 \Omega)$ as a characteristic volume of the packing of larger spheres, where $\Omega=3 \arccos (1 / 3)-\pi$ is the solid angle of the tetrahedron. $V_{p}$ is an estimation of the accessible volume of the smallest pore of the porous medium. Instead of the number $N$ of particles injected in the porous structure, we can now consider the ratio $N_{p}=V / V_{p}$, where $V=\frac{4}{3} \pi\left(\frac{d}{2}\right)^{3} N$. The mean transit time is deduced from the signal gathered at the output in the way detailed below.

\section{Experimental analysis}

The main phenomenon, which occurs inside the porous space, is lateral and transverse dispersion that is classically described as a Gaussian law for a fluid passing through a porous structure. Therefore, the evolution with time of the concentration of beads at the output follows an erf function, which is the solution of the convection-diffusion equation:

$$
\frac{\partial c(r, t)}{\partial t}+U \nabla c(r, t)=D_{/ /} \frac{\partial^{2} c(r, t)}{\partial x_{/ /}^{2}}+D_{\perp} \frac{\partial^{2} c(r, t)}{\partial x_{\perp}{ }^{2}}
$$

where $U$ is the interstitial velocity of the flow and $D_{/ /}$and $D_{\perp}$ are respectively the longitudinal and transversal diffusion coefficients [8]. Our weight scale will see the cumulative weight of falling beads, as shown in figure 2. We can already notice the difference with the best erf function fit drawn in the same figure. In others words, the

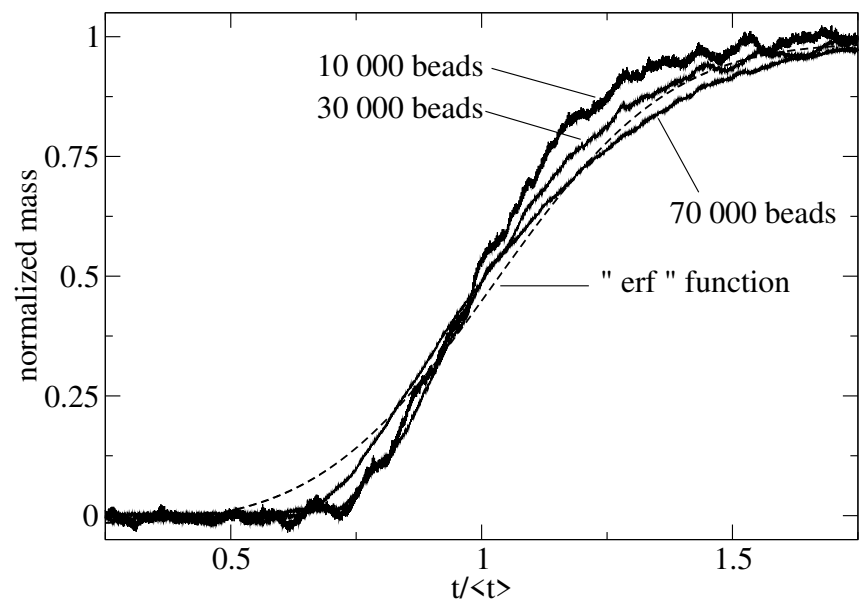

Figure 2. Evolution of the normalized mass of beads gathered at the output with normalized time for $N=70000, N=30000$ and $N=10$ 000. An adjustment with an erf function is also represented.

transit time distribution is not exactly a normal law. Indeed, if we focus our interest on the distribution of transit time, figure 3 demonstrates that distribution of residence time is a normal law with a tail for longer values of transit times. This behaviour reflects 
the appearance of lateness due to "trapping" of beads during the percolation process. The fraction of delayed particles becomes more significant when we increase the number of particles. This is why it is also possible to adjust our data by favouring shortest transit times. Such adjustments are shown in figure 4, on which we also clearly see the increase of the number of delayed particles with $N$. Nevertheless, as, in a first order

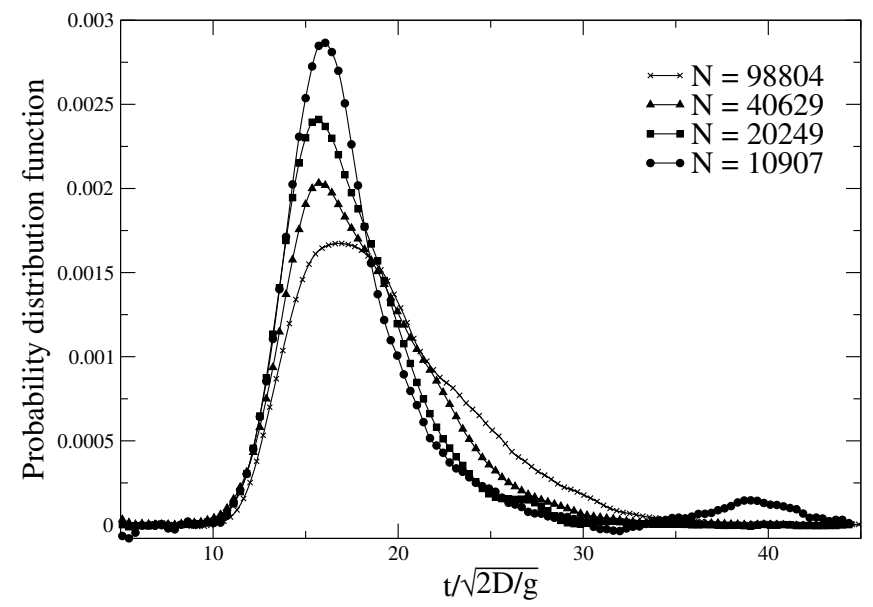

Figure 3. Distribution of transit times, normalized by free-fall time of a height of $D$, for different batch sizes with $D=16 \mathrm{~mm}, D / d=16$ and $H / D=10$.

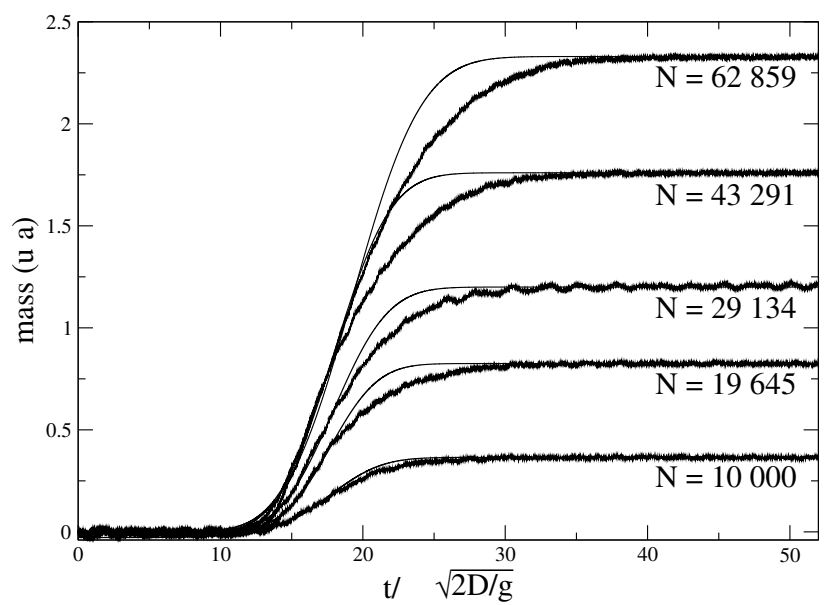

Figure 4. Cumulative mass with erf adjustment by giving less importance for shorter transit time for five values of $N . D=16 \mathrm{~mm}, D / d=16$ and $H / D=10$.

analysis, we are interested in the mean transit time determination, figure 2 shows that this Gaussian approximation can be used. Indeed, the difference in mean transit time determination between the two methods detailed above is small.

Using an erf adjustment, we have determined the mean transit time for different packing heights and for variable batches of small iron spheres ( $N$ between 1 and 100000 ). The porous medium is built with glass spheres of diameter $D=16 \mathrm{~mm}$. As we can see in figure 5, the residence time is proportional to the packing height even for different 
batch sizes. It can be related to previous results: for the case of a single particle falling down either in a 3D porous medium [5], or in a 2D system [9], the percolation velocity is constant. In fact, this is true if the size of the packing is large enough to minimize the influence of some transition regime at the beginning of the process. This stationary state appears for height higher than a threshold value, of the order of few large grain sizes, below which no granular temperature equilibrium can occur. We have not reported measurements of mean transit times for $H / D$ smaller than three to four large bead layers. This is for the reason described previously and because of strong differences in the transit time due to some time fluctuations of the beads, during the entering of the porous structure. Indeed, according to the possible presence of a pore under the opening hatch, a difference of one diameter distance can occur. For all these reasons, it is not possible to extrapolate the three curves to zero. Concerning figure 5, let us mention that the three curves are not parallel, which already suggests that the percolating velocity depends on the number of percolating particles. That is what we are going to see now, in more detail, in the next section.

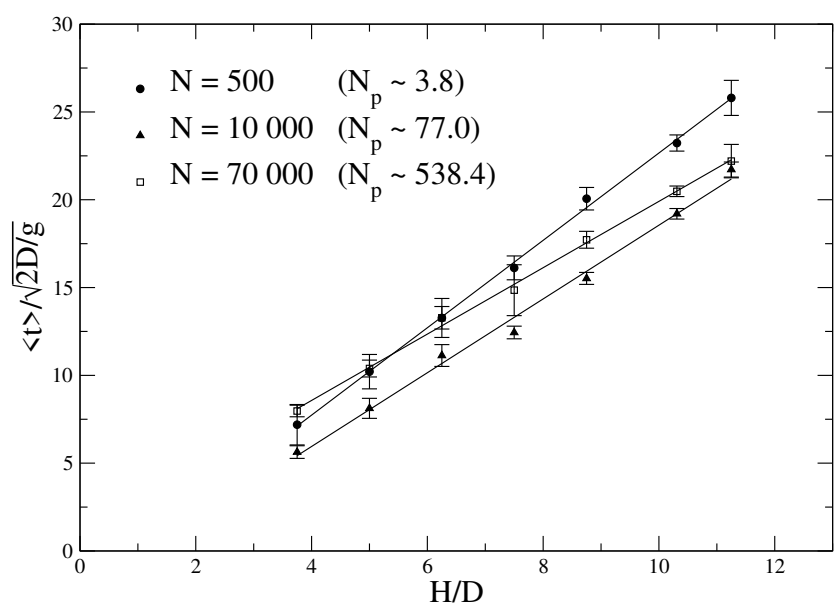

Figure 5. Dependence of the normalized mean transit time on $H / D$ for a launch of $N=500,10000,70000$ particles with $D=16 \mathrm{~mm}$ and $D / d=16$.

\section{Cooperative flow regime}

We have performed another set of experiments in order to study the impact of the number of percolating spheres on the mean transit time. It is evident that if only one particle falls down through a porous structure, it can explore the complete free space without any perturbation. However, this is no longer true if several particles fall at the same time. In this case, all these moving particles can interact one with another and also with the fixed ones. If we are near the critical value, which was defined previously and corresponds to the trapping threshold, only one or two particles can pass together through the same pore. If several particles are to transit the porous medium, they have to pass one after another. On the other hand, if we work far from the trapping 
threshold, a large number of them can pass simultaneously through the same pore. The mean residence time depends of the volume $N_{p} V_{p}$ occupied by the batch rather than size ratio only. So, mean transit time depends directly on the number $N$ of percolating particles.

We have plotted in figure 6 the evolution of the mean time with $N_{p}$ for a height of $H=10 D$. As mentioned previously, it is reasonable to think that the percolation velocity will decrease with the particle number due to pores jamming. In other words, the mean transit time would increase with $N_{p}$, due to the difficulty of the percolating particles finding accessible pores. Nevertheless, figure 6 shows that the phenomenon is more complex, and mainly we can define the existence of three flow domains.

In this study, we have two main effects of the spontaneous gravitational percolation: a significant rebound of a falling bead on fixed spheres (due to a large restitution coefficient) and also a large decrease of moving bead velocities due to the fact that the falling particles collide with each other and with the packing. In the first regime, from $N_{p}=1$ to approximately 50, the mean transit time decreases when the number of falling particles grows. As $N_{p}$ increases, they lose more and more energy by collisions with their neighbours. This implies that when $N_{p} \approx 50$ they are mainly falling down directly in the

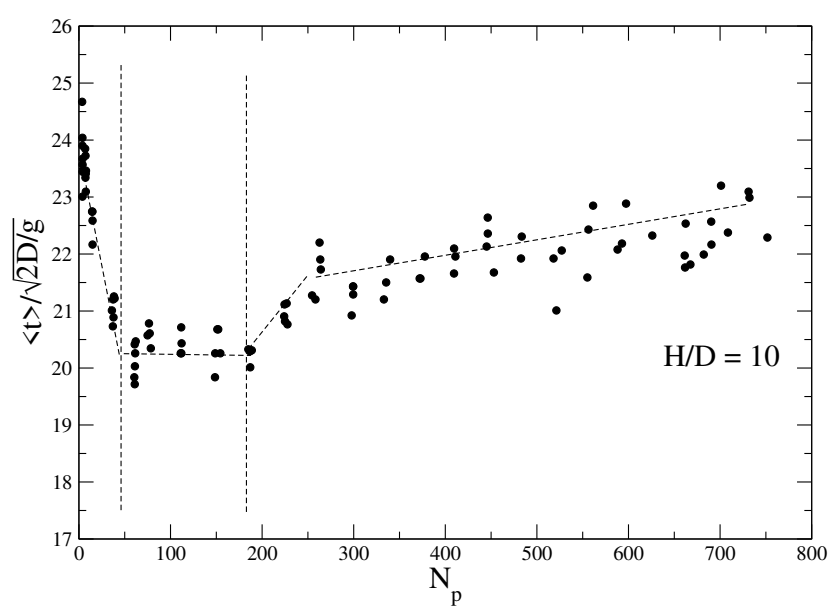

Figure 6. Dependence of the normalized mean transit time on $N_{p}$ for $D=16 \mathrm{~mm}$, $D / d=16$ and $H / D=10$.

lower pore structure, without bouncing around. As the number of small beads increases further, an equilibrium between the direct falling process and the inter-particle collision events is obtained and can be observed in a second domain from $N_{p} \approx 50$ to $\approx 200$. In this steady gravity regime, the mean percolation velocity is quite independent of $N_{p}$. So the mean transit time does not depend on $N$. If we continue to increase the number of falling beads, we observe a third regime, for $N_{p}>200$. The particle velocities slow down due to the crossing of a large number of particles simultaneously through the same pore. In fact, this regime can be divided into two sub-regimes. Firstly, from $N_{p} \approx 200$ to $\approx 250$, the dependence of the slowdown on $N_{p}$ is important. This behaviour reflects a beginning of a jamming effect of pores situated in the flow zone. The jamming effect 
grows with $N_{p}$ and then, for $N_{p}>250$, the increase of the mean transit time with $N_{p}$ is less important due to accumulation of particles in connecting pores. For such values of $N_{p}$, a pore jamming is related to the jamming of pores which are connected to it, by the obstruction of preferential paths. We can suppose that for $N_{p}>750$ we could observe a saturation of the mean transit time which would correspond to a total jamming of preferential paths.

Figure 7 represents the same evolution of the mean transit time for three packing heights. For $H / D=5$, the three regimes of flow are also visible. The second domain seems to appear for smaller number of particles than for $H / D=10$.

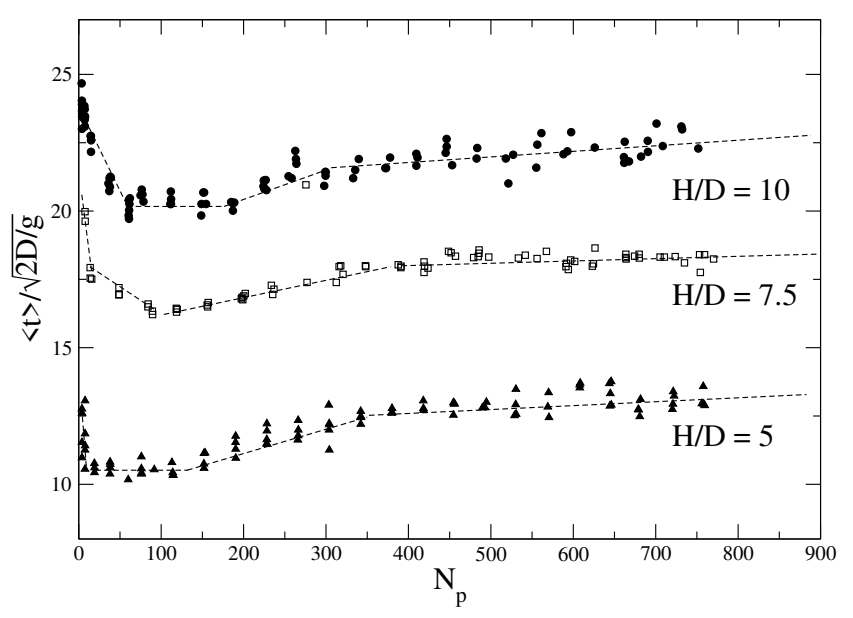

Figure 7. Dependence of the normalized mean transit time on $N_{p}$ for $D / d=16$ and $D=16 \mathrm{~mm}$. Three series of experiments, with different packing heights $H$, are represented.

\section{Numerical simulation}

\subsection{The numerical method}

In order to analyse influences of different geometrical and mechanical parameters, we couple experimental results with numerical simulations. We have chosen to make a model as close as possible to the real process which occurs when we launch a set of small particles through a pile made with larger spheres. Many algorithms exist to build a random 3D packing of mono-size spheres [10]. We have chosen to use the Powell's algorithm [11], as here constructed packings are built as if under gravity. Each sphere is placed in the lowest possible position, in contact with three spheres already placed. This leads to six contacts on average per sphere and porosity around 0.40 . Our porous structure is made by 70000 spheres of radius $D=20 \mathrm{~mm}$ inside a box of $70 D \times 70 D \times 175 D$ respectively in the $x, y$ and $z$ directions. To avoid finite size effects, the packing of larger spheres is generated with periodic boundary conditions in the $x$ and $y$ directions. A small size distribution of sphere radii (5\%) has been introduced in order to avoid local ordered zones and also to reproduce the natural dispersion size of 
a bead set. We build an upper box containing another packing of spheres of diameter d. This box is horizontally centred on top of the previous one and is almost cubic. To simulate the motion of large numbers of small spheres through a static packing of larger ones, we use the event-driven method [12] for the dynamic part of the simulation. In this approach, collisions are considered as instantaneous and binary; this means that only one collision can occur at a given time. The basic principle of this method consists in the resolution of the trajectory equation and by sorting the events. In spite of the periodicity of the porous medium, interactions between moving spheres do not take into account this periodicity (i.e. only spheres in the same periodic space can collide).

At the beginning of the simulation, the program calculates for each small sphere the shortest time interval from the present to a collision with one of its neighbours. Then, all these time intervals are sorted and the program jumps to the shortest collision time. Only positions of the two concerned spheres are evaluated, the event collision is computed and the program calculates the new velocities of the two spheres involved in this collision. After this, all the possible new collisions which could occur for these two spheres are computed and integrated in the sorting event tree. Then, the next event is treated and so on. The percolation process ends when all the small spheres have reached a height equal to their radii. In other words, this means that they touch the bottom of the packing.

In contrast to the experimental case, our simulations let us have access to the internal porous structure and more precisely to the displacement of particles. As shown in figure 8, we can follow the inner path of each moving bead from the entry to the output of the structure.

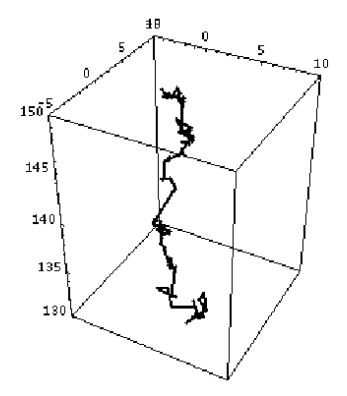

Figure 8. Zoom of a particle trajectory inside the box.

\subsection{Mono-sized spheres}

We have simulated experiments for different numbers $N$ of particles and so for different values of $N_{p}$. As we can observe in figure 9 , the mean transit time decreases with $N_{p}$ and seems to reach a constant value for $N_{p} \approx 140$. In the studied range of $N_{p}$, this behaviour is in agreement with experimental results presented previously.

In order to analyse the effect of the size ratio we have performed simulations dealing with $D / d$ and also with the restitution coefficient $e$. Figure 10 demonstrates that the mean 
transit time decreases with $D / d$. When the ratio $D / d$ is close to the trapping threshold, the residence time is quite long due to the difficulty of crossing the pore structure. A particle has to slow down and almost stop to cross each pore. When we increase the aspect ratio, the crossing is made easier and the mean transit time decreases. Figure 11 represents the dependence of the mean square displacement of particles, in a transversal direction, on time for different ratios $D / d$. It illustrates an increase of lateral dispersion when particles have smaller diameters. This explains why the decrease of the mean transit time, in figure 10, is less and less important with $D / d$. Moreover, we should notice that the linearity of the dependence of $\left\langle(\Delta x)^{2}\right\rangle$ on time illustrates the diffusive behaviour of the percolation process.

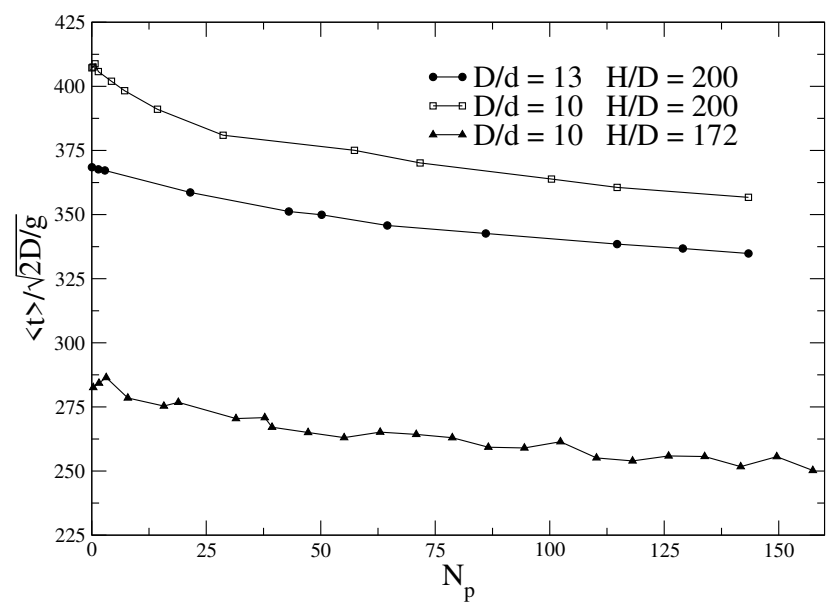

Figure 9. Evolution of the normalized mean transit time with the blob size $N_{p}$ for different values of size ratios $D / d$ and $H / D$.

Figure 10 shows also that the mean transit time increases with $e$ and confirms our assumption: for higher coefficients of restitution $e$, bounces of falling particles are more

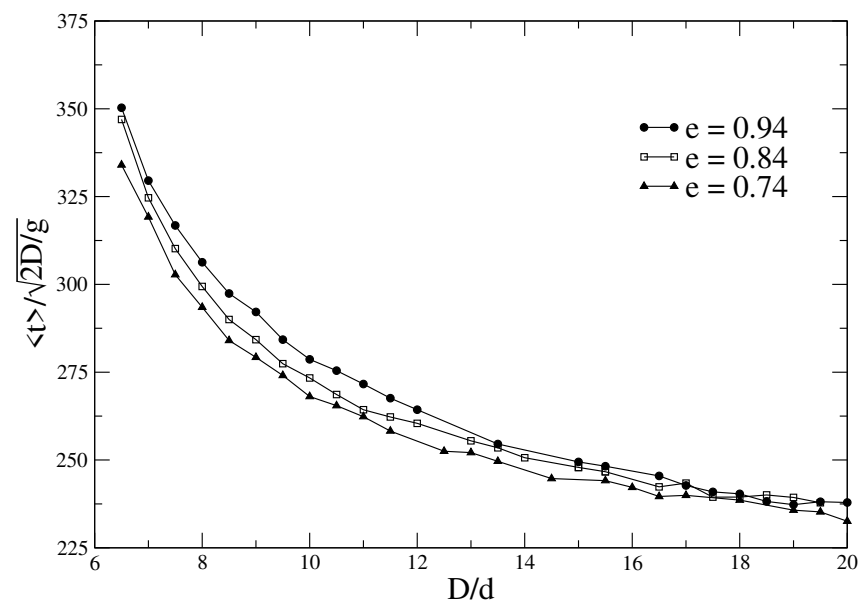

Figure 10. Normalized mean transit time evolution versus the size ratio $D / d$ for three coefficients of restitution with $N=1000$ and $H / D=172$. 


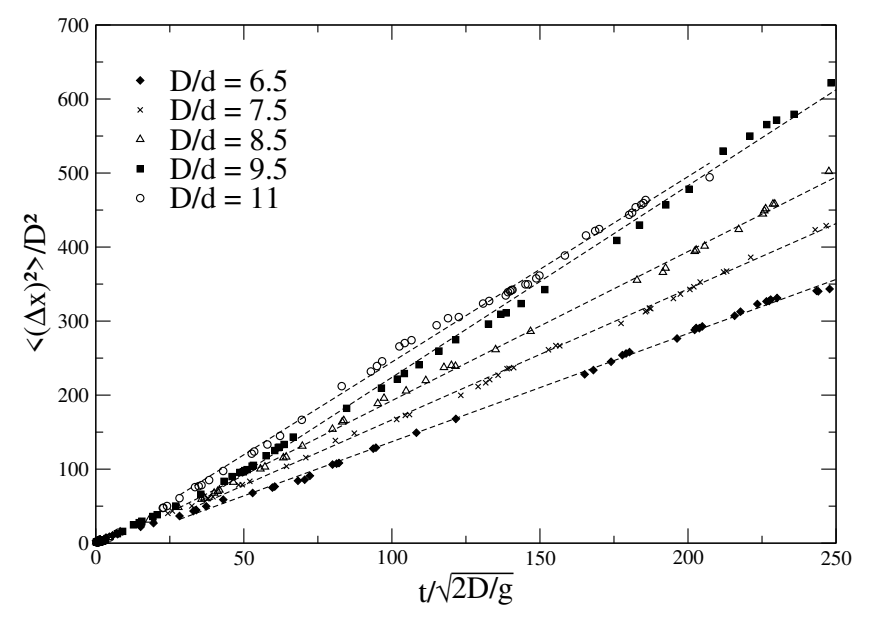

Figure 11. Dependence of $\left\langle(\Delta x)^{2}\right\rangle$ on normalized $t$ for a release of $N=1000$ particles and for different $D / d$.

important and exploration of the pore structure is made easier. The probability that a particle can almost go back to its previous altitude is not negligible. Figure 12 shows the linearity between the evolutions of mean variance of the position distribution in the $x$ direction with the mean altitude of the beads. It illustrates that dispersion is easier for higher restitution coefficient and implies, with comparison with figure 11, that the mean percolation velocity is constant after a transitional regime.

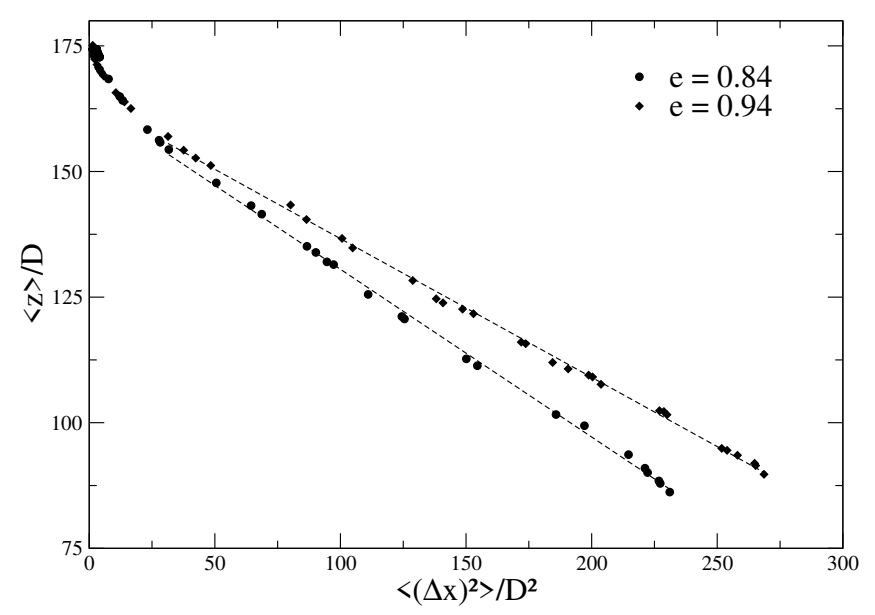

Figure 12. Mean altitude reached by the batch in function of mean square displacement in the $x$ direction for two restitution coefficients.

\subsection{Bi-disperse spheres}

We have carried out a preliminary series of simulations to extrapolate these behaviours to the case of two releases of particle batches with different diameters, in order to study the possibility of using such a process as a granular mixer. Let $d_{1}$ and $d_{2}$ be the diameters of particles of each set with $d_{1}>d_{2}$. These two piles are laterally centred at the top 
of the packing of larger spheres of diameter $D$ and the ratio between $d_{1} / d_{2}=1$. . The percentages of beads with diameters $d_{1}$ and $d_{2}$ are equal to $50 \%$. So we have $n_{1}=600$ particles of diameter $d_{1}, n_{2}=600$ of diameter $d_{2}$ and $N=n_{1}+n_{2}=1200$.

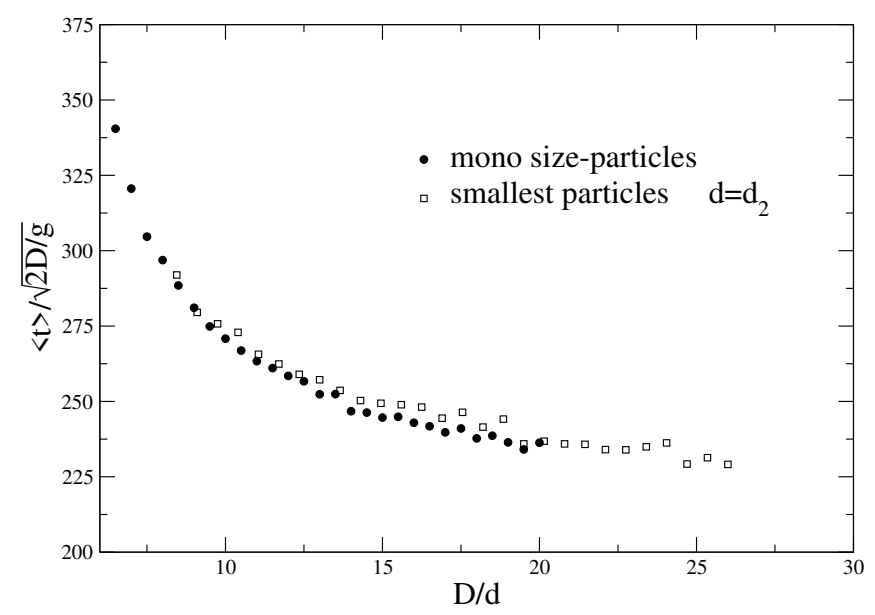

Figure 13. Evolution of normalized mean transit time for smallest particles of diameter $d_{2}$ with $D / d$ for a batch release of 1200 particles with $50 \%$ by number of each. Results for a simple mono-size launch of 1200 particles of diameter $d_{1}$ are also represented.

Figure 13 shows the evolution of mean transit time for the smaller particles versus the size ratio $D / d_{2}$. We have also represented the evolution of mean transit time in the case of a simple mono-size launch of 1200 particles. Figure 13 shows that particles of diameter $d_{2}$ behave almost like a set of 1200 particles made only with this size. The smallest particles do not see the influence of intermediate ones. More complementary investigations have to be made.

\section{Conclusions}

An experimental study of numerous particles flowing in a porous structure has been realized. The steady state, which is reached during the percolation process, shows us that vertical percolation velocity is constant. This behaviour is comparable to the case of a mono-particle flow previously studied. But collective effects, due to the transit of many particles in the structure, induce acceleration of the transit process and also jamming effects with particular consequences on the flow velocity, like the existence of flow regimes or delays compared to the fluid case. Despite these considerations, the separation between individual effects and collective ones is not trivial. Numerical simulations are performed to access the internal motion and to examine the influence of some physical parameters on the transit process. Moreover, it allows us to simulate mixing of particles with different physical properties, and leads us to think that the use of such a process could be helpful to obtain, in a cheaper way, homogeneous mixtures. 


\section{References}

[1] A. D. Rosato, K. J. Strandburg, F. Prinz, and R. H. Swendsen. Why the brazil nuts are on top: Size segregation of particulate matter by shaking. Phys. Rev. Lett., 58(10):1038, 1987.

[2] F. Cantelaube and Y. L. Duparcmeur. Geometrical analysis of avalanches in a 2d drum. J. Phys. I France, 5:581-596, 1995.

[3] J. A. Dodds. The porosity and contact points in multicomponent random sphere packings calculated by a simple statistical geometric model. J. Colloid Interface Sci., 77:317-327, 1980.

[4] J. Bridgwater, N. W. Sharpe, and D. C. Stocker. Particle mixing by percolation. Trans. Instn. Chem. Engrs., 47:114, 1969.

[5] L. Samson. Analyse de l'écoulement d'un grain dans des systèmes désordonnés bi- et tridimensionnels. PhD thesis, Université de Rennes1, 1997.

[6] I. Ippolito, L. Samson, and J. P. Hulin. Diffusion of a single particle in a 3d random packing of spheres. Eur. Phys. J. E, 3:227-236, 2000.

[7] L. Oger, J. P. Troadec, and B. Tharaud. Particle percolation through a porous medium. In J. Raper, editor, World Congress on Particle Technology 4, page 175, Sydney, july 2002.

[8] E. Charlaix. Dispersion en milieu poreux: mise en évidence de longueurs caractéristiques. $\mathrm{PhD}$ thesis, Université Paris 6, 1987.

[9] L. Bruno, A. Calvo, and I. Ippolito. Dispersive flow of diks through a two-dimensional galton board. Eur. Phys. J. E, 11:131, 2003.

[10] L. Oger, J. P. Troadec, A. Gervois, and N.N. Medvedev. Computer simulations and tesselation of granular materials. In J.F. Sadoc and N. River, editors, Foams and Emulsions, N 354, pages 527-546. NATO-ASI Series, Kluwer academic publishers, Dordrecht, 1999.

[11] M.J. Powell. Computer-simulated random packing of spheres. Powder Technology, 25:45-52, 1980.

[12] S. Luding, E. Clément, A. Blumen, J. Rajchenbach, and J. Duran. Studies of columns of beads under external vibrations. Phys. Rev. E, 49:1634, 1994. 